\author{
Anna Sulikowska \\ ORCID: 0000-0003-2939-3031 \\ Universität Szczecin, Szczecin
}

DOI: $10.19195 / 0435-5865.144 .18$

\title{
Embodiment und (Un-)Höflichkeit. Kognitive Aspekte kulturell geprägter Konzepte
}

\begin{abstract}
s
Eine der Grundprämissen der Kognitiven Linguistik bildet die Embodiment-These, die besagt, dass die menschliche Kognition und konzeptuelle Organisation sich aus der Art und Weise ergeben, in denen unsere artenspezifischen Körper mit der Umwelt interagieren. Das Fundament des konzeptuellen Systems eines Menschen baut also auf der Wahrnehmung, Sensomotorik, auf körperlicher und sozialer Erfahrung auf. Im folgenden Beitrag wird auf die Frage eingegangen, inwieweit kulturell geprägte Konzepte wie die Höflichkeit und Unhöflichkeit embodied sind. Der Analyse werden Idiome sowie figurative Einwortlexeme unterzogen.
\end{abstract}

Schlüsselwörter: konzeptuelle Metapher, image schemas, embodiment, figurative Sprache

\section{Embodiment and (im-)politeness: Cognitive aspects of culture-bound concepts}

One of the most important assumptions within the Cognitive Lingustics is the embodiment thesis, which postulates the human's cognition and conceptual organization being formed by the contact of the body with the environment. The basis for the human-specific conceptual system is the perception, sensomotorics and the physical and social experience. The article discusses the question of embodiment of the culture-based concepts like politeness and impoliteness. In the scope of the conducted analysis are idioms and figurative one-word lexems.

Keywords: conceptual metaphors, image schemas, embodiment, figurative language

Anna Sulikowska, Uniwersytet Szczeciński, Instytut Filologii Germańskiej, Al. Piastów 40b, bud. 5, pok. 103, 71-065 Szczecin, Polen, E-Mail: anna.sulikowska@usz.edu.pl

Received: 25.09.2018, accepted: 8.04.2019

Germanica Wratislaviensia 144, 2019

(C) for this edition by CNS 


\section{Einleitung}

Das Konzept der Höflichkeit/ Unhöflichkeit scheint auf den ersten Blick historisch-kulturell begründet zu sein. Die Höflichkeit ist unzertrennbar an gesellschaftliche Normen und soziale Umgangsformen gebunden, stark durch kulturelle Eigentümlichkeiten und historisches Wissen geprägt, wovon selbst der Ausdruck zeugt: Die etymologische Verbindung mit dem Nomen Hof ,fürstliche Residenz, fürstliches Gefolge und Milieu' ist hier offensichtlich und auch für Laien nachvollziehbar (vgl. mhd. hovelich ,dem Hof angemessen, fein gebildet', DWDS-Etymologie, Zugriff 22.09.2018). Die Motiviertheit für die Konzeptualisierungen von Höflichkeit/ Unhöflichkeit in der figurativen Sprache würde man folglich vor allem im kulturell-sozialen Bereich der menschlichen Erfahrung erwarten und suchen. Im vorliegenden Artikel wird der Frage nachgegangen, inwieweit die figurativen, übertragenen Konzeptualisierungen der Höflichkeit/ Unhöflichkeit in der körperlichen Erfahrung verankert, durch image schemas und konzeptuelle Metaphern bedingt sind. Einen theoretischen Hintergrund bildet dabei der holistische Ansatz der Kognitiven Linguistik und eine seiner zentralen Grundannahmen - die Embodiment-These.

\section{Grundprämissen der Kognitiven Linguistik}

Die Grundprämissen der Kognitiven Linguistik lassen sich in drei Punkten zusammenfassen (Evans/Green 2006: 27-50):

(i) Generalisation commitment geht davon aus, dass alle Subsysteme der Sprache wie Phonologie, Semantik, Syntax, allgemeinen kognitiven Regeln wie z.B. der Kategorisierung, Schematisierung oder Automatisierung unterliegen.

(ii) Cognitive commitment steht in Opposition zu Chomskys Sprachtheorie und hebt hervor, dass die Sprache kein Modul darstellt. Die Kognitive Linguistik ist holistisch und es wird postuliert, dass man die Sprache und die Bedeutungen nicht unabhängig von der Gesamtheit des menschlichen Wissens und der menschlichen Erfahrung auffassen kann.

(iii) Die Embodiment-These beruht auf der Annahme, dass die Kognition und Sprache weitgehend im Körper und in der körperlichen, perzeptiven, sensomotorischen und sozial-kulturellen Erfahrung verankert sind. „Mind is embodied, meaning is embodied and thought is embodied in the most profound sense. This is the substance of an embodied realism." (Johnson/Lakoff 2003: 249). Diese Prämisse der Kognitiven Linguistik wird im Folgenden fokussiert und es wird erklärt, was unter embodied realism (dt. Erfahrungsrealismus) und Embodiment verstanden wird. 


\section{Erfahrungsrealismus und Embodiment}

Embodied realism wird in Abgrenzung zum objektivistischen Realismus definiert. Um den innovativen Wert des Erfahrungsrealismus zu erklären, werden die beiden Perspektiven gegenübergestellt.

Die abendländische Vorstellung der Geisteswissenschaften beruhte Jahrhunderte lang auf zwei philosophischen Grundannahmen: dem Geist-Körper-Dualismus von René Descartes sowie einer bereits von Aristoteles eingeleiteten Doktrin im Rahmen der Epistemologie - dem objektivistischen Realismus. Realismus bedeutet, dass man die Existenz der reellen Welt voraussetzt. Das Attribut ,objektivistisch“ bringt zum Ausdruck, dass die reelle Welt mit Hilfe von objektiven, logischen Regeln erforscht und beschrieben werden kann. Das Denkvermögen und die Sprache sind demnach transzendent, d.h. von der Natur unseres Körpers unabhängig. Denken beruht auf der Manipulation von abstrakten Symbolen, die ihre Bedeutung durch Parallelen zu Entitäten und Kategorien der reellen Welt erhalten. Der Verstand widerspiegelt also die Natur, ${ }^{1}$ die Logik der externen Welt.

Diese Grundannahmen des objektivistischen Realismus wurden implizit von der Linguistik übernommen: In der traditionellen Sprachwissenschaft (im Strukturalismus, Generativismus) versuchte man die Sprache mit Hilfe von rigiden logisch-formalen Regeln zu beschreiben. Viele Aspekte der Sprache (z.B. im phonologischen, morphologischen oder syntaktischen Bereich) ließen sich auf diese Art und Weise erfolgreich auffassen, problematisch blieb allerdings immer die Bedeutungsfrage. ${ }^{2}$ Das etablierte Modell der Komponentenanalyse, das die Bedeutungen mit Hilfe von hinreichenden und notwendigen Merkmalen (die sog. binäre Merkmalssemantik, vgl. Löbner 2003: 201) beschreibt, ist zwar präzise und ökonomisch, lässt allerdings viele wichtige Aspekte der sprachlichen Realität (z.B.: Prototypikalitätseffekte, Verzahnung von sprachlichen und enzyklopädischen Bedeutungsaspekten, Verschwommenheit der Kategoriengrenzen, Einfluss von kontextuellen Faktoren auf die Bedeutungskonstituierung, Allgegenwärtigkeit der Polysemie und Ambiguität in der Sprache, Unmöglichkeit der Feststellung von kleinsten Bedeutungselementen) außer Acht. Die Bedeutungen sind zu komplex, um sich mit Hilfe von strikten, binären Merkmalen und rigiden Regeln erfassen zu lassen. Zur Auffassung der Bedeutung muss ein Modell vorgeschlagen werden, das die Präzision der traditionellen Semantik zugunsten einer größeren sprachlichen Realitätstreue aufgibt - an einem solchen Modell arbeitet die Kognitive Linguistik, während das philosophische Fundament des zu erarbeitenden Modells der embodied realism (Erfahrungsrealismus) bildet.

Die philosophischen Grundlagen des Erfahrungsrealismus wurden von Lakoff (1987) sowie Lakoff/Johnson (1999) gelegt. Auch hier wird angenommen,

1 Die Metapher von R. Rorty (1979).

2 Aus diesem Grunde lag die Semantik jahrelang am Rande des sprachwissenschaftlichen Interesses, oder wurde sogar explizit (z.B. von amerikanischen Strukturalisten) aus der Linguistik ausgeklammert. 
dass die reelle Welt existiert. Der Realismus ist aber embodied, d.h. es wird vorausgesetzt, dass die Konzepte und Konzeptualisierungen nicht transzendent sind, sondern durch die Beschaffenheit unseres Körpers und die körperliche und sozial-kulturelle Erfahrung beeinflusst werden.

Die Leithypothesen des Erfahrungsrealismus lassen sich nach Lakoff (1987: 162-163) folgendermaßen zusammenfassen:

(i) Der Verstand ist „kein Spiegel der Natur“ - die mentalen Repräsentationen der äußeren Reize stehen nie in der 1:1-Beziehung zu der externen Realität. Mentale, interne Repräsentationen haben immer einen konstruktiven Charakter, es sind Gebilde des menschlichen Geistes.

(ii) Kognition und Sprache sind an das denkende und sprechende Subjekt gebunden und können nicht unabhängig von ihm beschrieben werden, sie sind embodied. Die Strukturen, die unserem konzeptuellen System zugrunde liegen, sind weitgehend durch körperliche Erfahrung gestaltet, weswegen der Kern des konzeptuellen Systems eines Menschen direkt mit der Wahrnehmung, Körperbewegungen, körperlicher und sozialer Erfahrung zusammenhängt. „The cognition envisaged by cognitive linguists is noninsular, being grounded in perception and bodily experience“" (Langacker 2008: 28). Man kann also die Sprache nicht unabhängig von einem Menschen und seinen inhärenten körperlichen Eigenschaften beschreiben.

Unter Embodiment wird demnach eine These aufgefasst, dass unsere Kognition, unser konzeptuelles System und folglich auch die Sprache in der körperlichen Erfahrung verankert und durch diese Erfahrung bedingt sind. Die Grenzen zwischen der Perzeption, Kognition und Sprache werden aufgeweicht. Die Perzeption und damit senso-motorische Erfahrung bilden eine natürliche Grundlage der Kognition. Konzeptuelle Strukturen entstehen auf der Basis der perzeptuellen Erfahrung, manche (bei weitem aber nicht alle) von diesen konzeptuellen Strukturen werden herausgehoben und versprachlicht - sie bilden dann lexikalische Konzepte.

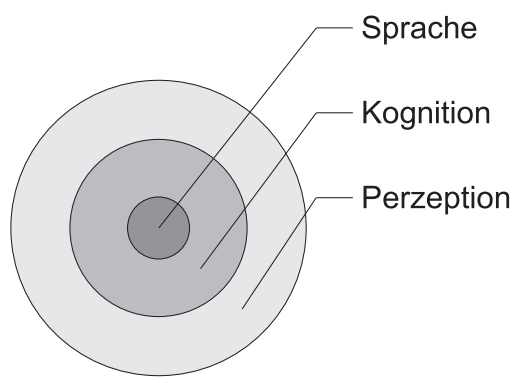

Abb. 1: Die fließenden Übergänge zwischen der Perzeption, Kognition und Sprache

Auch wenn Embodiment in der ersten Linie auf die Heraushebung und Beschreibung allgemeiner, universeller, wegen der Körperbeschaffenheit allen Menschen gemeinsamen Erfahrungen physischer Natur ausgerichtet ist, bedeutet dies nicht, dass kulturelle Aspekte durch das Radar der Kognitiven Linguistik nicht 
erfasst werden. Bereits in ihrem Standardwerk, den 1980 veröffentlichten Metaphors we live by weisen Lakoff und Johnson auf die grundlegende Rolle der weit gefassten Kultur in der Herausbildung des konzeptuellen Systems hin:

Das, was wir als „direkte physische Erfahrung“ bezeichnen, hängt niemals allein davon ab, daß wir einen Körper bestimmter Bauart haben; alle Erfahrung ereignet sich vielmehr in einem riesigen Kosmos kultureller Vorgaben. Deshalb kann es irreführend sein, wenn wir von direkter physischer Erfahrung sprechen, als ob es einen Kern der unmittelbaren Erfahrung gäbe, den wir dann von unserem Konzeptsystem her ,interpretieren“. Kulturell geprägte Annahmen, Wertvorstellungen und Einstellungen sind kein konzeptueller Überzug, den wir nach Belieben unserer Erfahrung überstülpen können oder auch nicht. Es wäre korrekter zu sagen, daß alle Erfahrung durch und durch kulturabhängig ist, daß wir unsere „Welt" in einer Weise erfahren, derzufolge die Erfahrung selbst unsere Kultur schon in sich trägt. (Lakoff/Johnson 2000 [1980]: 71)

Zugleich räumen sie allerdings ein:

Doch selbst wenn wir einräumen, dass in jeder Erfahrung „Kultur“ enthalten ist, können wir immer noch die wichtige Abgrenzung vornehmen zwischen den Erfahrungen, die „eher“ physischer Natur sind, wenn wir z.B. aufstehen, und den Erfahrungen, die „eher“ kultureller Natur sind, wenn wir z.B. an einer Hochzeitsfeier teilnehmen. (ebd.)

Embodiment kann demnach unterschiedlich weit aufgefasst werden, sich entweder auf die Verankerung unserer Kognition in der körperlichen Erfahrung sensu stricto, oder auf ihre Verankerung in der Gesamtheit unserer Erfahrung sensu largo beziehen. In den Fokus der Aufmerksamkeit des vorliegenden Beitrags wird die engere Auffassung gerückt.

\section{Embodiment, image schemas und konzeptuelle Metaphern}

Laut Embodiment-These widerspiegelt die Sprache nicht unmittelbar und objektiv die Natur, die äußere Welt, sondern eher ein unikales Konstrukt dieser Welt, unsere arten- und kulturspezifische Sicht auf die Welt. ${ }^{3}$ Diese Sicht ist demnach zum einen durch die Möglichkeiten des menschlichen Wahrnehmungsapparates eingeschränkt, zum anderen an artenspezifische Eigenschaften des menschlichen Körpers gebunden: Ein offensichtliches Beispiel für die Abhängigkeit unserer Kognition vom Wahrnehmungsapparat ist die Perzeption der Farben. Die Menschen nehmen nur einen Ausschnitt von dem Farbenspektrum wahr und - bei allen Unterschieden bei der Kategorisierung der Farben in unterschiedlichen Sprachen referieren wir vor allem auf diese Farben.

Auf einem abstrakteren Niveau beweisen Lakoff und Johnson, dass unser konzeptuelles System und die Sprache ebenfalls durch die artenspezifische Beschaffenheit des menschlichen Körpers beeinflusst werden. So findet Bipedie, d.h. die

3 ,Language does not directly reflect the world. Rather, it reflects our unique human construal of the world: our ,world view' as it appears to us through the lens of our embodiment". (Evans/Green 2006: 48) 
Tatsache, dass sich die Menschen auf zwei Beinen in einer senkrechten Position bewegen, ihre Widerspiegelung in der Urpolarität UNTEN-OBEN, die wohl allen Kulturen gemeinsam ist. Die aufrechte Körper-Orientierung unterscheidet uns von anderen Lebewesen: Oben ist der Kopf und die wichtigsten Sinnesorgane (Augen, Ohren, Nase, Mund), von oben sieht man mehr und man kann die Lage besser beurteilen, oben ist folglich positiv gewertet. Mit oben werden demnach Übersicht, Aufstieg, Kontrolle, positive Entwicklung, das edle Menschentum, Himmel, Geist, Gott assoziiert, in der Höhe, auf den Podien stehen Menschen in leitenden Positionen, auf den Thronen sitzen Fürsten und Könige, auf dem Olymp wohnen Götter, die Kirchtürme ragen in den Himmel, zur Siegerehrung gibt es Podeste. Unten liegt das Reich des Animalischen, Triebhaften, Primitiven, die Regression, der Hades, die Hölle. Solche Urpolaritäten haben ihren Ursprung in image schemas, den sog. Vorstellungsschemata, die im Folgenden näher erläutert werden.

Unter image schemas werden tief liegende kognitive Strukturen aufgefasst, die auf den rekurrenten senso-motorischen Erfahrungen basieren und in diesen Erfahrungen verankert sind.

Image schemas sind präkonzeptuell, sie tauchen in der frühesten kindlichen Erfahrung auf: Das Vorstellungsschema CONTAINER/CONTAINMENT (BEHÄLTER) wird möglicherweise bei der Nahrungsaufnahme herausgebildet: Das Baby nimmt wahr, dass sein Körper einem Behälter ähnlich ist, der gefüllt werden kann. Die Erfahrung der CONTAINER/CONTAINMENT-Relation ist in unserem Alltag omnipräsent: Wir erfahren unseren Körper als einen Behälter (indem wir uns z.B. den Bauch vollschlagen) sowie als Objekt in anderen Behältern, wenn wir z.B. in Armen gehalten werden, einen kleinen Raum verlassen oder in einen Bus einsteigen. Das Vorstellungsschema PATH (WEG) taucht möglicherweise schon während der Geburt auf: Es ist der erste Weg, auf dem man einen Ort verlässt und an einem anderen ankommt. Schon hier erfährt das Kind die interne Logik des Weg-Schemas: Um auf der Strecke den Endpunkt zu erreichen, muss man sich sequentiell, in der Zeit vom Punkt A zum Punkt B bewegen. Das image schemas BALANCE resultiert aus der Erfahrung der Schwerkraft, der Menschen als Erdenwesen ausgesetzt sind. Das image schema FORCE baut auf den ersten Erfahrungen der Interaktion mit der Welt auf.

Bei image schemas handelt es sich also um fundamentale Strukturen unserer Kognition, „,inkorporierte Muster sensomotorischer Körpererfahrungen“ (Jäkel 2003: 288). Image schemas sind dermaßen grundlegend und dabei so abstrakt, dass sie schwer erfassbar sind, allerdings stellen sie einen wichtigen Ankerpunkt für das ganze konzeptuelle System dar (vgl. Hampe 2005: 2). Hervorzuheben ist dabei der multimodale Charakter der image schemas: Die erste Komponente image (Bild) legt zwar nahe, dass es sich um bildhafte Strukturen handelt, es ist aber nicht der Fall. Image wird hier im psychologischen Sinne als imaginativ verwendet und bezieht sich auf alle sensomotorischen Systeme: den Seh-, Hör- und Tastsinn, die Propriozeption, d.h. Eigenempfindung: Lagesinn, Kraftsinn, Bewegungssinn; das motorische und vestibulare (mit Gleichgewicht verbundene) System. Auch wenn image schemas häufig als Wörter (РATH) oder Bilder dargestellt werden, stellen diese sym- 
bolischen Formen nur eine (die Kommunikation erleichternde) Vereinfachung dar: Es handelt sich um holistische mentale Repräsentationen perzeptueller Erfahrungen in unterschiedlichen Modalitäten (Johnson 1987: xx; Hampe 2005: 1).

Image schemas liegen konzeptuellen Metaphern zugrunde, so wie sie von Lakoff und Johnson beschrieben worden sind. Viele mächtige Metaphern von einem großen Generalitätsgrad, sog. bildschematische Metaphern (ein Terminus von Baldauf 1997: 123) bauen direkt auf image schemas auf. Unter konzeptuellen Metaphern werden feste, etablierte Mappings von einem kategorialen Charakter verstanden, die einen relativ konkreten, vielen Menschen gemeinsamen Erfahrungsbereich (Ausgangsdomäne) mit einem schwerer zu strukturierenden, abstrakteren Bereich (Zieldomäne) verbinden.

So liegt der konzeptuellen Metapher KÖRPER IST BEHÄLTER das image schema CONTAINER (BEHÄLTER) zugrunde, die Metapher selbst motiviert zahlreiche Spezifizierungen/Ausprägungen, die als konzeptuelle Metaphern von einem kleineren Generalitätsgrad betrachtet werden:

Tab. 1: Image schema BEHÄLTER, die darauf aufbauenden konzeptuellen Metaphern und Beispiele der sprachlichen Manifestationen

\begin{tabular}{|c|c|c|c|}
\hline $\begin{array}{l}\text { Image } \\
\text { schema }\end{array}$ & $\begin{array}{c}\text { Konzeptuelle } \\
\text { (bildschematische) } \\
\text { Metapher von einem } \\
\text { großen Generalitätsgrad }\end{array}$ & $\begin{array}{l}\text { Konzeptuelle Metaphern } \\
\text { von einem kleineren } \\
\text { Generalitätsgrad }\end{array}$ & $\begin{array}{l}\text { Beispiele für die } \\
\text { Manifestationen } \\
\text { in der Sprache }\end{array}$ \\
\hline \multirow[t]{3}{*}{$\begin{array}{l}\text { CONTAINER } \\
\text { (BEHÄLTER) }\end{array}$} & \multirow[t]{3}{*}{$\begin{array}{c}\text { KÖRPER } \\
\text { IST BEHÄLTER }\end{array}$} & $\begin{array}{c}\text { GEFÜHLE UND } \\
\text { GEDANKEN SIND DER } \\
\text { INHALT DES BEHÄLTERS }\end{array}$ & $\begin{array}{l}\text { jdn. mit Angst erfüllen, } \\
\text { von Neid erfüllt sein, } \\
\text { fast vor Wut zerbersten, } \\
\text { außer sich vor Freude sein, } \\
\text { seine Wut an jmdm. } \\
\text { /etw. auslassen/ in sich } \\
\text { hineinfressen, } \\
\text { aufgestaute Wut, } \\
\text { Wut im Bauch haben }\end{array}$ \\
\hline & & $\begin{array}{l}\text { WISSEN IST INHALT DES } \\
\text { BEHÄLTERS }\end{array}$ & $\begin{array}{c}\text { sich etw. aneignen, } \\
\text { jmdm. nicht in den Kopf } \\
\text { [hinein]gehen/[hinein]wollen, } \\
\text { Stroh im Kopf haben, } \\
\text { ein Nürnberger Trichter, } \\
\text { etw. begreifen, } \\
\text { die Weisheit nicht gerade mit } \\
\text { Löffeln gefressen haben }\end{array}$ \\
\hline & & $\begin{array}{l}\text { HAUT IST DIE WAND DES } \\
\text { BEHÄLTERS } \\
\text { EMPFINDLICHKEIT IST } \\
\text { EINE DÜNNE WAND DES } \\
\text { BEHÄLTERS, } \\
\text { UNEMPFINDLICHKEIT IST } \\
\text { EINE DICKE WAND DES } \\
\text { BEHÄLTERS }\end{array}$ & $\begin{array}{c}\text { dickes Fell haben, } \\
\text { dickfällig sein, } \\
\text { dünne Haut haben, } \\
\text { dünnhäutig sein, } \\
\text { robust, abgehärtet, } \\
\text { widerstandsfähig sein, } \\
\text { jmdn. mit Samthandschuhen } \\
\text { anfassen, } \\
\text { zerbrechlich sein }\end{array}$ \\
\hline
\end{tabular}


Dasselbe bezieht sich auf das Vorstellungsschema PATH (WEG): Die Konzeptualisierungen unseres Lebens (auch der Phylo- und Ontogenese), der Moral, der Karriere, der Schwierigkeiten im Leben, des Fortschritts sind weitgehend durch das Vorstellungsschema WEG und die konzeptuelle Metapher LEBEN IST EIN WEG strukturiert:

Tab. 2: Image schema WEG, die darauf aufbauenden konzeptuellen Metaphern und Beispiele der sprachlichen Manifestationen

\begin{tabular}{|c|c|c|c|}
\hline $\begin{array}{l}\text { Image } \\
\text { schema }\end{array}$ & $\begin{array}{c}\text { Konzeptuelle } \\
\text { (bildschematische) } \\
\text { Metapher } \\
\text { von einem großen } \\
\text { Generalitätsgrad }\end{array}$ & $\begin{array}{l}\text { Konzeptuelle Meta- } \\
\text { phern } \\
\text { von einem kleineren } \\
\text { Generalitätsgrad }\end{array}$ & $\begin{array}{l}\text { Beispiele für die } \\
\text { Manifestationen } \\
\text { in der Sprache }\end{array}$ \\
\hline \multirow[t]{3}{*}{$\begin{array}{l}\text { WEG } \\
(\mathrm{PATH})\end{array}$} & LEBEN IST EIN WEG & $\begin{array}{l}\text { GEBURT IST ANFANG } \\
\text { DES WEGES, TOD IST } \\
\text { ENDE DES WEGES }\end{array}$ & $\begin{array}{c}\text { auf die Welt kommen, } \\
\text { zur Welt bringen, } \\
\text { aus dem Leben scheiden/gehen, } \\
\text { aus dem Leben/unserer Mitte geris- } \\
\text { sen werden, } \\
\text { dahingehen/hinübergehen/ } \\
\text { davongehen, } \\
\text { den Weg alles Fleisches gehen, } \\
\text { seinen letzten Gang/seine letzte } \\
\text { Reise antreten, } \\
\text { in die Ewigkeit eingehen, } \\
\text { die Welt verlassen }\end{array}$ \\
\hline & & $\begin{array}{c}\text { SCHWIERIGKEITEN SIND } \\
\text { HINDERNISSE } \\
\text { AUF DEM WEG }\end{array}$ & $\begin{array}{c}\text { Steine in den Weg legen, } \\
\text { eine Hürde nehmen, } \\
\text { jmdm. etwas aus dem Weg räumen/ } \\
\text { schaffen, } \\
\text { jmdm. Hindernisse/ einen Hemm- } \\
\text { schuh in den Weg legen, } \\
\text { jmdm. in den Weg kommen/ treten/ } \\
\text { laufen, } \\
\text { jmdm. den Weg versperren/ ver- } \\
\text { treten, } \\
\text { jmdm. in die Quere kommen/ gera- } \\
\text { ten/ laufen/ rennen, } \\
\text { die Klippen umfahren/ umschiffen, } \\
\text { in eine Sackgasse geraten/ sich in } \\
\text { eine Sackgasse verrennen/ in einer } \\
\text { Sackgasse stecken, } \\
\text { jmdn./ etw. in ausweglose Lage } \\
\text { bringen/ in einer ausweglosen Lage } \\
\text { (sein) }\end{array}$ \\
\hline & & $\begin{array}{l}\text { FORTSCHRITT IST EINE } \\
\text { VORWÄRTSBEWEGUNG }\end{array}$ & $\begin{array}{c}\text { neue/andere Wege beschreiten/ } \\
\text { gehen, } \\
\text { den Horizont öffnen, } \\
\text { ein Schritt vorwärts, } \\
\text { auf der Strecke bleiben, } \\
\text { große Sprünge machen }\end{array}$ \\
\hline
\end{tabular}




\section{Konzeptualisierungen der Höflichkeit/Unhöflichkeit in der figurativen Sprache}

In der folgenden Untersuchung versuche ich anhand der Konzeptualisierungen der HÖFLICHKEIT/UNHÖFLICHKEIT in der figurativen Sprache, darunter im phraseologischen Bestand des Deutschen der Frage nachzugehen, inwieweit die Konzepte HöFLICHKEIT/UnHÖFLICHKEIT embodied sind, d.h. inwieweit ihre Konzeptualisierungen in der körperlichen Erfahrung verankert, durch image schemas und konzeptuelle Metaphern bedingt sind.

$\mathrm{Zu}$ diesem Zweck wurde anhand onomasiologisch und semasiologisch ausgerichteter Nachschlagewerke, der phraseologischen und allgemeinen Wörterbücher des Deutschen (DUW online, DWDS-Wörterbuch, Redensarten-Index, Müller 2005, Schemann 2012) eine Liste figurativer Spracheinheiten erstellt. Es wurden dann die konzeptuellen Metaphern und die ihnen zugrunde liegenden image schemas eruiert, die zur Versprachlichung der HÖFLICHKEIT/UNHÖFLICHKEIT als einer attributiven Eigenschaft eines Menschen herangezogen werden. Erwartungsgemäß übersteigt die Anzahl der negativ konnotierten Spracheinheiten die Anzahl der Phraseologismen, die höfliches Verhalten und Höflichkeit zum Ausdruck bringen. Die ermittelten konzeptuellen Metaphern stellt das Schema (2) dar:

\section{jd. ist höflich / unhöflich}

BEHÄLTER

KÖRPER IST BEHÄLTER

(UN) HÖFLICHKEIT IST DIE BESCHAFFENHEIT

DER ÄUßEREN FORM DES BEHÄLTERS

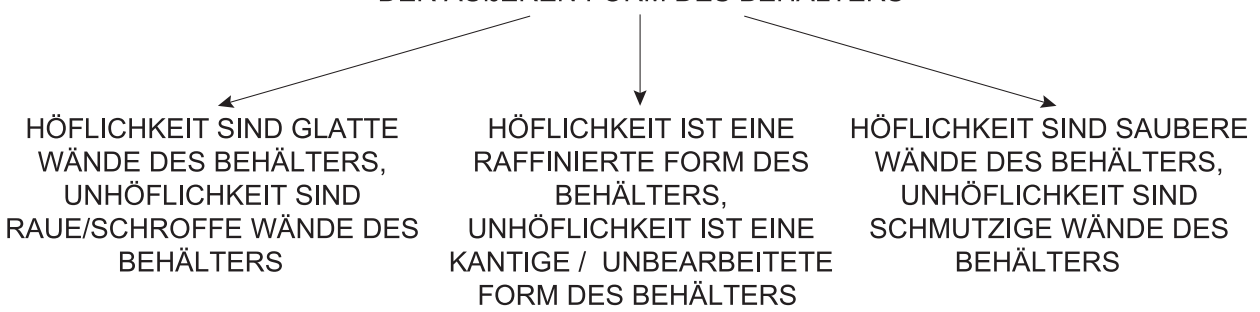

Abb. 2: Konzeptuelle Metaphern, die den Diskursbereich Höflichkeit/Unhöflichkeit als attributive Eigenschaft eines Menschen strukturieren

Wie ersichtlich, spielt die wichtigste Rolle bei der Konzeptualisierung der HÖFLICHKEIT/UnHÖFLICHKEIT als Charaktereigenschaft einer Person das image schema BEHÄLTER. Die metaphorischen Übertragungen beziehen sich vor allem auf strukturelle Elemente des Behälters: Innen, Außen und Grenze, d.h. seine Wände und eben die Wände des Behälters werden bei den Konzeptualisierungen der HÖFLICHKEIT/UNHÖFLICHKEIT hervorgehoben, profiliert. 
So wie das Äußere des Behälters, seine Beschaffenheit, Sauberkeit, Form auf seinen Inhalt schlussfolgern lassen, so geben in der metaphorischen Übertragung auch die Umgangsformen eines Menschen den ersten Eindruck von seiner Natur. Höflichkeit, vornehme Umgangsformen werden als glatte, saubere, sorgfältig geschliffene Wände konzeptualisiert; unhöfliches, freches Benehmen konzeptualisieren wir unter Rückgriff auf seine schroffe, unbearbeitete, schmutzige Form. Es lassen sich dabei drei Ausprägungen der bildschematischen Metapher KÖRPER IST BEHÄLTER ermitteln.

\section{(I) HÖFLICHKEIT SIND GLATTE WÄNDE DES BEHÄLTERS UNHÖFLICHKEIT SIND RAUE, SCHROFFE WÄNDE DES BEHÄLTERS}

Die beiden konzeptuellen Metaphern manifestieren sich in folgenden Idiomen sowie figurativen Einwortlexemen:

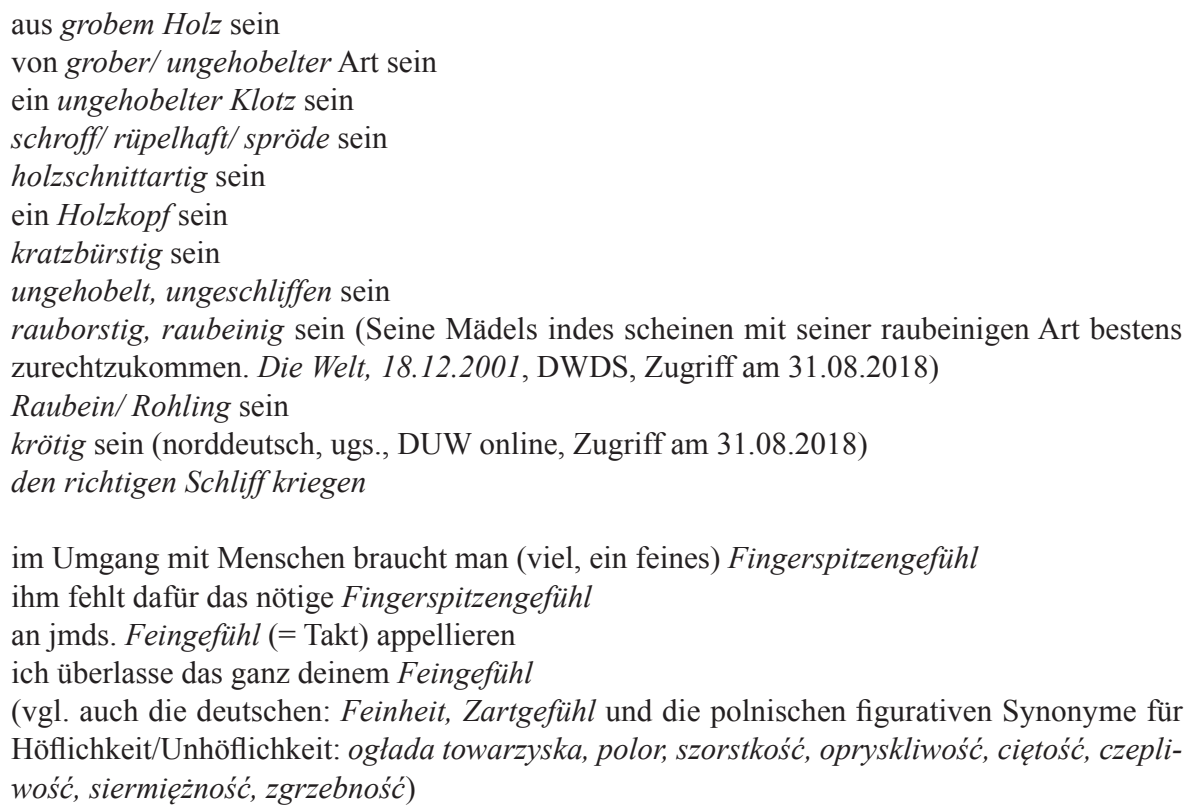

Die körperliche Verankerung der Konzeptualisierungen ist hier offensichtlich: Im taktilen Bereich wird das Glatte, Weiche, Warme positiv konnotiert, an dem Schroffen, Harten, Ungeschliffenen kann man sich leicht verletzen. (Kalte, harte Gegenstände werden mit Waffen: Schwertern, Messern, Pistolen assoziiert, an rauen, unebenen Flächen oder Gegenständen kann man sich zerkratzen oder schmerzhafte Holzsplitter davontragen). Glatte, d.h. keine sichtbaren, spürbaren Unebenheiten aufweisende Flächen leisten einen geringeren Widerstand. Dies 
wird auf die Domäne der Aufgaben/Herausforderungen übertragen die Sache/die Arbeit geht glatt vonstatten, eine glatte Landung, ein glatter Bruch in der Medizin (DUW online, Zugriff 2.09.2018), die taktilen Empfindungen werden aber auch in die Domäne der Eigenschaften eines Menschen projiziert: Wer/was glatt ${ }^{4}$ ist, ist so beschaffen, dass er/es keine Komplikationen, Schwierigkeiten, Unannehmlichkeiten bereitet.

Bemerkenswert ist dabei, dass die Adjektive der Unglätte: rau, roh, grob, spröde, ungehobelt, ungeschliffen ${ }^{5}$ zwei Lesarten aufweisen: Die Tendenz, die taktile Empfindung der Unglätte mit der Domäne der sozialen Umgangsformen zu mappen ist im Deutschen so stark ausgeprägt, dass sich keins der genannten Wörter ausschließlich auf die körperliche Wahrnehmung bezieht.

\section{(II) HÖFLICHKEIT IST EINE RAFFINIERTE FORM DES BEHÄLTERS UNHÖFLICHKEIT IST EINE KANTIGE/UNBEARBEITETE FORM DES BEHÄLTERS}

Während das erstgenannte Metaphernpaar den taktilen Wahrnehmungsbereich fokussiert, werden in dem zu besprechenden Fall zusätzlich die visuellen Aspekte zu der Konzeptualisierung von HöFLICHKEIT herangezogen. Die ungeschliffenen, klobigen Formen eines unbearbeiteten Holzklotzes oder Felsbrockens werden metaphorisch auf den Bereich der Unhöflichkeit übertragen. So wie die Bearbeitung eines Rohmaterials zu einer raffinierten Form arbeits- und zeitaufwändig ist, so resultiert auch die Kultiviertheit, Feinheit, Höflichkeit aus einem langwierigen Erziehungsprozess. Die Umgangsformen muss man nämlich einem Kind anerziehen, einen Menschen kann man schleifen, ihm Schliff beibringen. Auf die alltägliche Erfahrung, aber auch den symbolischen Status des Kreises und der Kugel ist ebenfalls die positive Bewertung der runden Form zurückzuführen: Etwas

${ }^{4}$ Die Bedeutung des Adjektivs glatt wird ebenfalls erweitert und auf die Domäne der Höflichkeit/ Unhöflichkeit übertragen. Interessanterweise ist diese Teilbedeutung dennoch negativ konnotiert, vgl. DUW online, Zugriff am 21.09.2018: glatt ,4. allzu gewandt, übermäßig höflich; einschmeichelnd [und dabei unaufrichtig, heuchlerisch]'. Die Motiviertheit dieser Übertragung ist auf epistemisches Wissen zurückzuführen: Allzu glatte Flächen bieten keinen Halt. Da die Menschen von Natur aus nicht vollkommen sind, veranlasst uns die übertriebene Höflichkeit (Glätte) zur Vorsicht.

${ }^{5}$ Vgl. DUW online, Zugriff am 21.09.2018: rau ,1. auf der Oberfläche kleine Unebenheiten, Risse o. Ä. aufweisend, sich nicht glatt anfühlend, 4. im Umgang mit anderen Feingefühl vermissen lassend'; roh ,2.a unbearbeitet, unverarbeitet, (abwertend) anderen gegenüber gefühllos und grob, sie körperlich oder seelisch verletzend'; grob ,1.a in seiner Beschaffenheit derb, stark, 4.a (abwertend) im Umgangston mit anderen Menschen ohne Feingefühl, barsch und unhöflich'; spröde, 1.a hart, unelastisch und leicht brechend oder springend, 1.b. sehr trocken und daher nicht geschmeidig und leicht aufspringend, 3.a schwer zugänglich, abweisend, verschlossen wirkend'; ungehobelt ,1. nicht mit einem Hobel bearbeitet, geglättet, 2.a (abwertend) grob, rüde, unhöflich'; ungeschliffen,1. nicht geschliffen, 2. (abwertend) ohne gute Manieren, das rechte Taktgefühl im Umgang mit anderen vermissen lassend". 
abrunden wird mit ,vervollkommnen' gleichgesetzt, die runde Sache stellt eine reibungslose, gelungene Angelegenheit dar. Das Gegensatzpaar der konzeptuellen Metaphern manifestiert sich in folgenden sprachlichen Ausdrücken:

jemand mit Ecken und Kanten, jemand mit unangenehmen charakterlichen Eigenarten; ein Mensch, dessen Verhalten nicht den Erwartungen entspricht; ein streitbarer/eigenwilliger Mensch; jemand, der nicht leicht im Umgang, aber ehrlich ist ${ }^{`}$ (Redensarten-Index, Zugriff am 31.08.2018)

ohne Ecken und Kanten sein

ein eckiges Wesen haben

sich eckig benehmen

ein kantiger Charakter, Typ ,ein eigenwilliger, nicht leicht zugänglicher Charakter, Typ“ (DUW online, Zugriff am 31.08.2018)

klobig sein

ein Klotz sein

grobschlächtig sein

vierschrötig sein,,(besonders von Männern) von breiter, kräftiger, gedrungener Gestalt [und dabei derb-ungehobelt wirkend]' (DUW online, Zugriff am 31.08.2018)

ein Grobian, Rohling, Rowdy, Kloben sein

Auf einen groben Klotz gehört ein grober Keil, Wer grob/unverschämt ist, den muss man auch grob behandeln' (Redensarten-Index, Zugriff am 31.08.2018)

fein, formgewandt sein

gute Umgangsformen haben

umgänglich sein

den richtigen Schliff kriegen

(vgl. auch die polnischen figurativen Synonyme für Höflichkeit/Unhöflichkeit: sznyt, szlif towarzyski, układność, wyrobienie towarzyskie, nieokrzesanie, grubiańskość, gruboskórność, prostactwo, kanciastość, prymitywność, siermiężność, zgrzebność, przaśność)

\section{(III) HÖFLICHKEIT SIND SAUBERE WÄNDE DES BEHÄLTERS UNHÖFLICHKEIT SIND SCHMUTZIGE WÄNDE DES BEHÄLTERS}

In der dritten Ausprägung der bildschematischen Metapher KÖRPER IST BEHÄLTER wird die Höflichkeit mittels der sauberen Wände, die Unhöflichkeit unter Rückgriff auf die unangenehme Erfahrung des Anfassens von schmutzigen Gegenständen konzeptualisiert. Dies manifestiert sich in den Äußerungen:

pampig sein ,1. breiig, wie Pampe, 2. in grober Weise frech, patzig' ein pampiger Kellner (DUW online, Zugriff am 31.08.2018)

patzig sein ,1. in ungezogener Weise unwillig auffahrend, mit einer groben Antwort reagierend; unverschämt 2. klebrig, verschmiert' (DUW online, Zugriff am 31.08.2018)

ein ungewaschenes Maul haben, frech reden; vorlaut sein“ (Redensarten-Index, Zugriff am 31.08.2018)

(?) einen Ton am Leib (haben) ${ }^{6}$

${ }^{6}$ Ton weist zwei Bedeutungen auf: 1. vom Gehör wahrgenommene gleichmäßige Schwingung, 2. besonders zur Herstellung von Töpferwaren verwendetes lockeres, feinkörniges Sediment von gelblicher bis grauer Farbe. (DUW online, Zugriff am 31.08.2018) 
Ein besonderer, symbolischer Status kommt dabei den Ausscheidungsprodukten und Körperflüssigkeiten zu: Bei der Konzeptualisierung der Unhöflichkeit, eines unverschämten Benehmens wird grundsätzlich auf (umgangssprachliche, z.T. regionale) Bezeichnungen des Nasenschleims (z.B. Schnodder) zurückgegriffen:

frech wie [der] Rotz [am Ärmel] ,salopp: außerordentlich frech“ (Redensarten-Index, Zugriff, 31.08.2018)

rotzfrech sein ,sehr frech sein' (DUW online, Zugriff am 31.08.2018)

rotznäsig sein, (besonders von Kindern) [ungepflegt, schmutzig und] ungezogen und frech ${ }^{c}$ schnodderig/schnoddrig sein ,respektlos/unverschämt/angeberisch/lässig/provokativ/spöttisch auftreten/reden` (Redensarten-Index, Zugriff am 31.08.2018)

ein Schnösel sein ,junger Mann, dessen Benehmen als frech, ungezogen, überheblich empfunden wird' (Redensarten-Index, Zugriff am 31.08.2018)

\section{Zusammenfassung}

Die Embodiment-These besagt, dass unsere konzeptuellen Strukturen weitgehend durch die körperlichen, artenspezifischen Erfahrungen bedingt sind. Die durchgeführte Analyse der lexikalisierten metaphorischen Einwortlexeme sowie der Phraseologismen, mit denen die HÖFLICHKEIT/UNHÖFLICHKEIT konzeptualisiert wird, bestätigen diese Annahme zu einem erheblichen Grad: Auch wenn die Konzepte der HÖFLICHKEIT/UNHÖFLICHKEIT auf den ersten Blick kulturell begründet zu sein scheinen - Höflichkeit ist durch gesellschaftliche Umgangsformen geprägt - haben ihre metaphorischen Konzeptualisierungen doch in vielen Fällen eine körperlich erfahrbare Basis: Der Mehrheit der figurativen Spracheinheiten dieses Diskursbereiches liegt die bildschematische Metapher KÖRPER IST BEHÄLTER und ihre Ausprägung: (UN)HÖFLICHKEIT IST DIE BESCHAFFENHEIT DER ÄUSSEREN FORM DES BEHÄLTERS zugrunde. Keiner der genannten Metaphern lassen sich sechs Idiome zuordnen: ein situativ motivierter phraseologischer Vergleich:

frech wie Oskar (salopp: auf eine dreiste, kecke Art frech; Herkunft ungeklärt; vielleicht nach Oskar Blumenthal [1852-1917], der sehr scharfe und »freche« Kritiken schrieb) (DUW online, Zugriff am 31.08.2018)

sowie fünf Idiome und figurative Einwortlexeme, deren Motiviertheit auf allgemein-kulturelles Wissen zurückzuführen ist:

nicht die feine englische Art sein ,unhöflich sein, sich schlecht/ unfair/ unehrenhaft benehmen; etwas nicht den allgemeinen Regeln entsprechend handhaben' (Redensarten-Index, Zugriff am 31.08.2018)

bärbeißig sein ,brummig-unfreundlich` sein (DUW online, Zugriff am 31.08.2018)

bullenbeißerisch sein ,brummig-unfreundlich' sein (DUW online, Zugriff am 31.08.2018)

krachledern sein Krachlederne: ,zur bayrischen Tracht gehörende kurze Lederhose', krachledern: ,eine derb-heftige Art aufweisend' (DUW online, Zugriff am 31.08.2018)

eine gute Kinderstube/ keine gute Kinderstube haben 
Zusammenfassend lässt sich also in Bezug auf die zu untersuchenden sprachlichen Einheiten Folgendes feststellen:

We have a species-specific view of the world due to the unique nature of our physical bodies. In other words, our construal of reality is likely to be mediated in large measure by the nature of our bodies. (Evans/Green 2006: 45)

\section{Literatur}

\section{Quellen}

DUW online $=$ Duden online. https://www.duden.de.

DWDS = Das digitale Wörterbuch der deutschen Sprache. http://www.dwds.de.

Müller 2005 = Müller, Klaus (2005): Lexikon der Redensarten. Herkunft und Bedeutung deutscher Redewendungen. München.

Redensarten-Index $=$ www.redensarten-index.de.

Schemann 2012 = Schemann, Hans (2012): Synonymwörterbuch der deutschen Redensarten. 2. Aufl. Berlin/Boston.

\section{Sekundärlitaratur}

Baldauf, Christa (1997): Metapher und Kognition. Grundlagen einer neuen Theorie der Alltagsmetapher. Frankfurt am Main.

Evans, Vyvyan / Green, Melanie (2006): Cognitive Linguistics: An Introduction. Edinburgh.

Hampe, Beate (2005): Image Schemas in Cognitive Linguistics: Introduction. In: Hampe, Beate (Hrsg.): From Perception to Meaning: Image Schemas in Cognitive Linguistics. Berlin/New York. S. 1-12.

Jäkel, Olaf (2003): Wie Metaphern Wissen schaffen. Die kognitive Metapherntheorie und ihre Anwendung in Modell-Analysen der Diskursbereiche Geistestätigkeit, Wirtschaft, Wissenschaft und Religion. Hamburg.

Johnson, Mark (1987): The Body in the Mind: The Bodily Basis of Meaning, Imagination, and Reason. Chicago.

Johnson, Mark / Lakoff, George (2002): Why Cognitive Linguistics require Embodied Realism. In: Cognitive Linguistics 13(3), S. 245-263.

Lakoff, George (1987): Woman, Fire, and Dangerous Things. What Categories Reveal about the Mind. Chicago/London.

Lakoff, George / Johnson, Mark (1980): Metaphors We Live by. Chicago. Deutsche Ausgabe (2000): Leben in Metaphern: Konstruktion und Gebrauch von Sprachbildern. Aus dem Amerikanischen übersetzt von A. Hildenbrand. 2. korrigierte Aufl. Kempten.

Lakoff, George / Johnson, Mark (1999). Philosophy in the Flesh: The Embodied Mind and its Challenge to Western Thought. New York.

Langacker, Ronald W. (2008): Cognitive Grammar: A Basic Introduction. Oxford.

Löbner, Sebastian (2003): Semantik. Eine Einführung. Berlin/New York.

Rorty, Richard (1979): Philosophy and the Mirror of Nature. Princeton. 